\title{
Diabetic Foot Infection: Causative Pathogens and Empiric Antibiotherapy Considerations-The Experience of a Tertiary Center
}

The International Journal of Lower Extremity Wounds $1-7$

C) The Author(s) 2019

Article reuse guidelines: sagepub.com/journals-permissions DOI: 10.1 I77/15347346/9839815 journals.sagepub.com/home/ijl @SAGE

\author{
José Miguel Neves, MD', Bruno Duarte, MD', Margarida Pinto, MD², \\ Ana Formiga, $M^{3}$, and José Neves, $M^{3}$
}

\begin{abstract}
Most moderate-to-severe diabetic foot infections (DFIs) require hospitalization with urgent surgical approach and administration of empiric antibiotherapy. To ensure optimal antibiotic coverage, regular microbiological background updates are imperative. The purpose is to characterize the microbiological profile and the antibiotic sensitivity pattern of the DFI causative pathogens isolated within a specialized DFI unit of a tertiary hospital, in order to establish evidence-based policies regarding empirical antibiotic use. A cross-sectional study was conducted. Microbiological cultures and corresponding antibiotic sensitivity tests collected from moderate-to-severe DFls as a first approach to the hospitalized patient were retrieved and analyzed during a I2-month period. Two groups were analyzed: inpatients that had been previously followed at the diabetic foot clinic of the hospital and inpatients without a previous contact with the hospital services. A total of I 25 isolates obtained from 87 patients were deemed for analysis. Globally, a predominance of Gram-positive bacteria was observed (60\%). Staphylococcus aureus was the most common pathogen. The global ratio of methicillin-sensitive $S$ aureus to methicillin-resistant S aureus (MRSA) was I.3:I, with similar findings in both groups. According to the antibiotic sensitivity test results, and within the recommended empiric antibiotic regimens for DFI, piperacillin/tazobactam seems to be the most suitable option. Gram-positive bacteria prevail as the main isolates in DFls. Screening for MRSA-specific risk factors is mandatory. When going for a first empiric therapy, piperacillin/tazobactam is recommended in this institution, and an anti-MRSA agent should be added early, if necessary. We encourage continuous monitoring for the bacterial prevalence in Portuguese diabetic foot centers as it is paramount for the decision making regarding DFI protocols.
\end{abstract}

\section{Keywords}

diabetic foot ulcers, wound infection, tissue biopsy

Diabetes mellitus (DM), one of the most prevalent chronic diseases worldwide, continues to increase significantly. ${ }^{1}$ It is expected that by 2045 about 642 million people will be affected by this multisystemic disease. ${ }^{2}$ The number of DM patients in Portugal is also rising steadily, with an estimated prevalence of $13.3 \%^{3}$ in 2015. Diabetic foot ulcers (DFUs) are one of the major complications of this disease and occur in about $25 \%$ of the diabetic population. The infection of a DFU is a complex and costly medical disorder, with a high level of immediate and long-term morbidity. ${ }^{4}$ Diabetic foot infections (DFIs) account for up to one quarter of all diabetic hospitalizations in both Europe and the United States, making it the single most common reason for DM-related hospital admissions. The relative immunosuppressed environment and poor peripheral perfusion observed in diabetic patients creates opportunity for aggressive polymicrobial infections that often spread, either to the nearby tissues leading to gangrene or by a systemic route leading to sepsis, organ failure, or even death. In addition, the pathogens isolated in DFI have been showing increasingly higher antibiotic resistance rates. ${ }^{5,6}$ Unsurprisingly, the presence of an infection is associated with a 5-year mortality of around

\footnotetext{
'Department of Dermatology and Venereology, Centro Hospitalar Universitário de Lisboa Central.

${ }^{2}$ Microbiology Department, Centro Hospitalar Universitário de Lisboa Central.

${ }^{3}$ Diabetic Foot Unit, General Surgery Department, Centro Hospitalar Universitário de Lisboa Central.

Corresponding Author:

José Miguel Neves, Dermatovenereology Department, Hospital de Santo António dos Capuchos, Centro Hospitalar Universitário de Lisboa Central, Alameda Santo António dos Capuchos, Lisboa II69-050, Portugal.

Email: josemoneves@gmail.com
} 
$40 \%$, and accounts for the largest number of nontraumatic lower extremity amputations. ${ }^{\text {? }}$

A proper treatment strategy for moderate-to-severe DFIs encompasses both the use of empirical broad-spectrum antibiotics - tailored to the local bacteriological profile, severity of infection, and patient's comorbidities - and early surgical drainage and debridement. The former entails the need to study the microbiological flora and susceptibility of DFI pathogens in a particular center-based population. In this sense, we aimed to define the microbiological and antibiotic resistance profiles of the hospitalized patients in our specialized DF unit in order to establish evidence-based policies regarding empirical antibiotic use to treat DFIs. The final purpose was to cover the most common pathogens, while avoiding the unwarranted economic, biologic, and public health costs of the misuse of broad-spectrum antibiotics.

\section{Materials and Methods}

A cross-sectional study was conducted. Data extraction was performed by scrutinizing the electronic medical records of all patients hospitalized in a DF unit of a tertiary medical center, in the period between January 1, 2017, and December 31, 2017. Enrolled patients were analyzed as 2 independent cohorts. The first, named Outpatient Group (OG), included inpatients previously followed at the outpatient clinic of the hospital. The other, the External Group (EG), included patients with no previous history of DFU treatments at our hospital (mainly patients admitted directly from the emergency room or after a single first evaluation at the outpatient clinic).

The presence of a clinically infected foot ulcer localized below the malleolar process of a patient with DM was the main inclusion criteria. As per our center's protocol, DFIs are defined clinically (and not microbiologically) based on the presence of at least 2 classic symptoms or signs of inflammation (erythema, warmth, tenderness, pain, or induration) or purulent secretions. ${ }^{8,9}$ In accordance with the international guidelines, hospitalization was considered for moderate-to-severe infection cases (grades 3/4 accordingly to the PEDIS score). ${ }^{10,11}$

All samples collected from clinically infected DFUs, either by swab or surgical methods, were reviewed. As per our DF unit protocol, to avoid commensal flora isolates, all samples were collected by an experienced physician after ulcer rinsing with saline water and gentle debridement of superficial debris. Collected data consisted of isolated bacteriological specimens and respective in vitro antibiotic susceptibility and resistance profile. Standard processing methods for culture and antibiotic susceptibility test (AST) were employed at the microbiology laboratory department. ASTs were routinely performed for all microbiological samples. The anaerobic culture was not performed due to
Table I. Isolated Microorganisms.

\begin{tabular}{lrrr}
\hline Pathogens & OG & EG & Total \\
\hline Gram-positive & 25 & 50 & 75 \\
Corynebacterium striatum & 2 & 0 & 2 \\
Enterococcus spP & 2 & 13 & 15 \\
MSSA & 8 & 17 & 25 \\
MRSA & 8 & 11 & 19 \\
Staphylococcus lugdunensis & 1 & 0 & 1 \\
Streptococcus spP & 4 & 9 & 13 \\
Gram-negative & 26 & 24 & 50 \\
Achromobacter xylosoxidans & 1 & 0 & 1 \\
Acinetobacter baumaani & 1 & 2 & 3 \\
Enterobacter spP & 4 & 1 & 5 \\
Escherichia coli & 2 & 5 & 7 \\
Klebsiella spP & 3 & 1 & 4 \\
Morganela morganii & 3 & 0 & 3 \\
Proteus spp & 1 & 6 & 7 \\
Pseudomonas aeruginosa & 8 & 7 & 15 \\
Serratia marcescens & 1 & 2 & 3 \\
Stenotrophomonas maltophilia & 2 & 0 & 2 \\
Total & $5 \mathrm{I}$ & 74 & 125 \\
\hline
\end{tabular}

Abbreviations: OG, outpatient group; EG, external group; MSSA, methicillin-sensitive Staphylococcus aureus; MRSA, methicillin-resistant Staphylococcus aureus.

lack of standard procedures for handling anaerobic samples. "Polymicrobial" cultures, without isolation of a specific microorganism, were excluded by the microbiology laboratory.

\section{Results}

A total of 125 microbial isolates were obtained from the microbiological analyses of 87 samples - an average of 1.44 pathogens per culture $(38 \%[\mathrm{n}=33]$ contained more than 1 microbiological isolate, of which $32 \%[n=28]$ and $6 \%[\mathrm{n}=5]$ accounted for 2 and 3 pathogens, respectively). About $85 \%$ of the cultures were performed from surgical samples, while the remaining were obtained from swab of deep wounds.

Out of the total episode number (87), $72 \%$ (63) were male and $29 \%$ (24) were female patients. The average patient age was 67 years (maximum 94 years; minimum 31 years).

The OG was composed of 32 subjects, 25 males and 7 females (3.6:1), and this group accounted for a total of 51 microbiological isolates. The EG was composed of 55 subjects, 35 males and 17 females (2:1), accounting for a total of 74 bacterial isolates.

The detailed distribution of the isolates is presented in Table 1. A total of $60.0 \%(n=75)$ of the isolates were Gram-positive bacteria and 40\% $(n=50)$ were Gramnegative bacteria (1.5:1 ratio). Staphylococcus aureus $(\mathrm{n}=44)$ was the most common Gram-positive aerobe bacteria, 
Table 2. Resistance Profile of the Most Common and Resistant Isolated Pathogens.

\begin{tabular}{|c|c|c|c|c|c|c|c|c|c|}
\hline & $\begin{array}{c}\text { MSSA } \\
(\mathrm{N}=25)\end{array}$ & $\begin{array}{c}\text { MRSA } \\
(N=19)\end{array}$ & $\begin{array}{c}\text { Pseudomonas } \\
\text { aeruginosa } \\
(\mathrm{N}=15)\end{array}$ & $\begin{array}{l}\text { Enterococcus } \\
\operatorname{spp}(N=15)\end{array}$ & $\begin{array}{l}\text { Streptococcus } \\
\operatorname{spp}(N=13)\end{array}$ & $\begin{array}{l}\text { Proteus spp } \\
(\mathrm{N}=7)\end{array}$ & $\begin{array}{l}\text { Escherichia coli } \\
\qquad(\mathrm{N}=7)\end{array}$ & $\begin{array}{l}\text { Enterobacter } \\
\text { spp. }(\mathrm{N}=5)\end{array}$ & $\begin{array}{l}\text { Klebsiella spp } \\
\quad(\mathrm{N}=4)\end{array}$ \\
\hline Penicillin & $84 \%(2 \mathrm{I} / 25)$ & $100 \%(19 / 19)$ & & & & & & & \\
\hline Oxacillin & $0 \%(0 / 25)$ & $100 \%(19 / 19)$ & & & & & & & \\
\hline Ampicillin & & & & $7 \%(1 / 15)$ & $0 \%(0 / 13)$ & & & & \\
\hline $\begin{array}{l}\text { Amoxicillin/clavulanic } \\
\text { acid }\end{array}$ & & & & & & $43 \%(3 / 7)$ & $71 \%(5 / 7)$ & $100 \%(5 / 5)$ & $50 \%(2 / 4)$ \\
\hline $\begin{array}{l}\text { Piperacillin/ } \\
\text { tazobactam }\end{array}$ & & & $33 \%(5 / 15)$ & & & $0 \%(0 / 7)$ & $29 \%(2 / 7)$ & $0 \%(0 / 5)$ & $50 \%(2 / 4)$ \\
\hline Cefuroxime & & & & & & $43 \%(3 / 7)$ & $57 \%(4 / 7)$ & & $25 \%(1 / 4)$ \\
\hline Ceftazidime & $0 \%(0 / 25)$ & & $20 \%(3 / 15)$ & & & & $43 \%(3 / 7)$ & & $25 \%(1 / 4)$ \\
\hline Cefotaxime & & & & & & $0 \%(0 / 7)$ & $57 \%(4 / 7)$ & $0 \%(0 / 5)$ & $25 \%(I / 4)$ \\
\hline Meropenem & & & $20 \%(3 / 15)$ & & & $0 \%(0 / 7)$ & $0 \%(0 / 7)$ & $0 \%(0 / 5)$ & $0 \%(0 / 4)$ \\
\hline Ertapenem & & & & & & & $0 \%(0 / 7)$ & $0 \%(0 / 5)$ & $0 \%(0 / 4)$ \\
\hline Gentamycin & $0 \%(0 / 25)$ & $11 \%(3 / 19)$ & $13 \%(2 / 15)$ & & & $0 \%(0 / 7)$ & $43 \%(3 / 7)$ & $0 \%(0 / 5)$ & $0 \%(0 / 4)$ \\
\hline Tetracycline & $0 \%(0 / 25)$ & $11 \%(3 / 19)$ & & & & & & & \\
\hline Amikacin & & & $0 \%(0 / 15)$ & & & & $0 \%(0 / 7)$ & $0 \%(0 / 24)$ & \\
\hline Ciprofloxacin & & & $53 \%(8 / 15)$ & & & & $100 \%(7 / 7)$ & & \\
\hline Cotrimoxazol & $8 \%(2 / 25)$ & $6 \%(2 / 19)$ & & & $0 \%(0 / 13)$ & & & & \\
\hline Vancomycin & & $0 \%(0 / 19)$ & & $0 \%(0 / 15)$ & & & & & \\
\hline ESBL & & & & & & & $57 \%(4 / 7)$ & & \\
\hline
\end{tabular}

Abbreviations: MSSA, methicillin-sensitive $S$ aureus; MRSA, methicillin-resistant $S$ aureus; ESBL, extended spectrum $\beta$-lactamases.

followed by Enterococcus spp $(\mathrm{n}=15)$ and Streptococcus $\operatorname{spp}(\mathrm{n}=13)$. Within $S$ aureus isolates, $57 \%(\mathrm{n}=25)$ were methicillin-sensitive $S$ aureus (MSSA) and 43\% $(\mathrm{n}=19)$ were methicillin-resistant $S$ aureus (MRSA). The 5 most isolated Gram-negative organisms were Pseudomonas aeruginosa $(\mathrm{n}=15)$, Proteus spp $(\mathrm{n}=7)$, Escherichia coli $(\mathrm{n}=7)$, Enterobacter $\operatorname{spp}(\mathrm{n}=5)$, and Klebsiella $\operatorname{spp}(\mathrm{n}=4)$.

The predominance of Gram-positive bacteria was observed in the EG (2.1:1 ratio). When considering the OG, a similar prevalence of Gram-positive and Gram-negative bacteria was found, with a correspondent ratio of 0.96:1. S aureus was the most common isolate in both groups. The global ratio of MSSA-MRSA was 1.3:1, with similar findings when looking into the 2 groups separately.

AST results are described in Table 2. The included microorganisms were selected according to their relevance (quantity and resistance profiles). From this analysis, we highlight the resistance of Gram-negative bacteria to amoxicillin/clavulanic acid $(>40 \%)$. Even the broad-spectrum, parenteral, anti-pseudomonal antibiotic piperacillin/ tazobactam recorded rates of $29 \%, 33 \%$, and $50 \%$ resistance within E coli, P aeruginosa, and Klebsiella spp, respectively. An optimistic finding is the low resistance rate shown by the MRSA isolates to cotrimoxazol, both in OG and EG.

A comparison was drawn between the 2 groups on the pathogens' sensitivity to 3 usually employed empiric antibiotics (amoxicillin/clavulanic acid, piperacillin/tazobactam, and ertapenem), and data are depicted in Table 3.

\section{Discussion}

Proper empirical antibiotic selection is controversial as, to date, no empirical antimicrobial regimen has been shown to be superior in DFI treatment. ${ }^{12}$ A systematic analysis undertaken by the Iberoamerican Cochrane Center concluded that the evidence for the relative effects of different systemic antibiotics for the treatment of DFI is extremely heterogeneous and generally at unclear or high risk of bias. $^{8}$

The most well-established guidelines for the management of DFI are from Western countries where Grampositive bacteria, especially MSSA, are the most common pathogens in DFI. Empiric antibiotic regimens are usually chosen to cover those agents. The option to cover MRSA should rely on its local prevalence, on the infection severity, and on the comorbidities and risk factors of the patient. The establishment of MRSA risk factors is still a controversial theme, with a wide range of studies performed on the subject. The most commonly reported in DFI are recent hospitalization, antibiotic usage in the 6 months prior to the hospitalization, a chronic ulcer ( $\geq 4$ weeks), ulcer size $\geq 4 \mathrm{~cm}^{2}$, bone involvement, and previous history of MRSA infection or colonization. ${ }^{13-16}$ On the other hand, empiric antibiotic coverage of $P$ aeruginosa, according to the International Working Group on the Diabetic Foot guidelines, should be considered in certain scenarios, where its prevalence is increased, such as long-standing DFUs, severe DFIs of hospitalized patients, and those who received prior cycles of antibiotics. ${ }^{16-18}$ 
Table 3. Empiric Antibiotic Comparison Accordingly to the Pathogens Isolated.

\begin{tabular}{|c|c|c|c|c|c|}
\hline Pathogens & Isolates (n) & Isolates (\%) & $A C$ & PT & $\mathrm{E}$ \\
\hline \multicolumn{6}{|l|}{ External group } \\
\hline MSSA & 17 & $23 \%$ & $0 \%(0 / 17)$ & $0 \%(0 / 17)$ & $0 \%(0 / 17)$ \\
\hline MRSA & 11 & $15 \%$ & - & - & - \\
\hline Enterococcus spp & 13 & $18 \%$ & $0 \%(0 / 13)$ & $0 \%(0 / 13)$ & - \\
\hline Streptococcus spp & 9 & $12 \%$ & $0 \%(0 / 13)$ & $0 \%(0 / 13)$ & $0 \%(0 / 13)$ \\
\hline F Gram-negative & 15 & $20 \%$ & $60 \%(9 / 15)$ & $7 \%(1 / 15)$ & $0 \%(0 / 15)$ \\
\hline Pseudomonas aeruginosa & 7 & $9 \%$ & - & $29 \%(2 / 7)$ & - \\
\hline Gram NNF & 2 & $3 \%$ & $100 \%(2 / 2)$ & $100 \%(2 / 2)$ & $100 \%(2 / 2)$ \\
\hline Total & 74 & $100 \%$ & $39 \%(29 / 74)$ & $21 \%(16 / 74)$ & $45 \%(33 / 74)$ \\
\hline \multicolumn{6}{|l|}{ Outpatient group } \\
\hline MSSA & 8 & $15.7 \%$ & $0 \%(0 / 8)$ & $0 \%(0 / 8)$ & $0 \%(0 / 8)$ \\
\hline MRSA & 8 & $15.7 \%$ & - & - & - \\
\hline Enterococcus spp & 2 & $3.9 \%$ & $50 \%(\mathrm{I} / 2)$ & $50 \%(1 / 2)$ & - \\
\hline Streptococcus spp & 4 & 7.8 & $0 \%(0 / 13)$ & $0 \%(0 / 13)$ & $0 \%(0 / 13)$ \\
\hline Other Gram-positive & 3 & $5.9 \%$ & $0 \%(0 / 3)$ & $0 \%(0 / 3)$ & $0 \%(0 / 3)$ \\
\hline F Gram-negative & 15 & $29.4 \%$ & $87 \%(13 / 15)$ & $20 \%(3 / 15)$ & $0 \%(I I)$ \\
\hline Pseudomonas aeruginosa & 8 & $15.7 \%$ & - & $37.5 \%(3 / 8)$ & - \\
\hline Gram NNF & 3 & $5.9 \%$ & $100 \%(3 / 3)$ & $100 \%(3 / 3)$ & $100 \%(3 / 3)$ \\
\hline Total & 51 & $100 \%$ & $65 \%(33 / 5 I)$ & $35 \%(|8 / 5|)$ & $4 \mid \%(2|/ 5|)$ \\
\hline \multicolumn{6}{|l|}{ Global } \\
\hline MSSA (25) & 25 & $20 \%$ & $0 \%(0 / 25)$ & $0 \%(0 / 25)$ & $0 \%(0 / 25)$ \\
\hline MRSA (19) & 19 & $15.20 \%$ & - & - & - \\
\hline Enterococcus spp (15) & 15 & $12 \%$ & $7 \%(1 / 15)$ & $7 \%(1 / 15)$ & - \\
\hline Streptococcus spp (13) & 13 & $10.40 \%$ & $0 \%(0 / 13)$ & $0 \%(0 / 13)$ & $0 \%(0 / 13)$ \\
\hline Other Gram-positive & 3 & $2,40 \%$ & $0 \%(0 / 3)$ & $0 \%(0 / 3)$ & $0 \%(0 / 3)$ \\
\hline F Gram-negative & 30 & $24 \%$ & $73 \%(22 / 30)$ & $13 \%(4 / 30)$ & $0 \%(0 / 30)$ \\
\hline Pseudomonas aeruginosa & 15 & $12 \%$ & - & $33 \%(5 / 15)$ & - \\
\hline NF Gram-negative & 5 & $4 \%$ & $100 \%(5 / 5)$ & $100 \%(5 / 5)$ & $100 \%(5 / 5)$ \\
\hline Total & 125 & $100 \%$ & $50 \%(62 / 125)$ & $27 \%(34 / 125)$ & $43 \%(54 / 125)$ \\
\hline
\end{tabular}

Abbreviations: AC, amoxicillin/clavulanic-acid; PT, piperacillin/tazobactam; E, ertapenem; MSSA, methicillin-sensitive S aureus; MRSA, methicillinresistant $S$ aureus; F, fermenter; NF, non-fermenter.

According to the 2012 Infectious Disease Society of America (ISDA) guidelines on antibiotic treatment of a moderate-to-severe DFI, the main agents to consider are second- and third-generation cephalosporines, combination between $\beta$-lactamic antibiotics and $\beta$-lactamase inhibitors and carbapenems, with possible additions when facing specific circumstances. ${ }^{10}$ The broad-spectrum antibiotics intensively compared in DFI clinical trials are ertapenem, ampicillin/sulbactam, imipenem/cillastin, and piperacillin/ tazobactam. The Portuguese Directorate-General of Health recommends the administration of carbapenems or ureidopenicillins with a $\beta$-lactamase inhibitor (piperacillin/tazobactam) for severe infections. ${ }^{19}$ It is our opinion that these therapeutic options must be adjusted accordingly to the microbiological profile of each unit.

In Portugal, few studies have been performed to describe the microbiological profiles of DFIs. To our knowledge, there are 3 studies concerning this topic: Cabete et $\mathrm{al}^{4}{ }^{4}$ Mendes et $\mathrm{al},{ }^{7}$ and Barbosa et al. ${ }^{20}$ In both Mendes et al and Barbosa et al studies a Gram-positive predominance was observed, similar to other Western studies. Cabete et al's study included all the microbiological isolates collected from DFUs in outpatients and inpatients of our Diabetic Foot Multidisciplinary unit. ${ }^{4}$ While portraying the microbiological profile present in our unit activity, information about empiric antibiotic regimens could not be withdrawn.

Gram-positive bacteria, particularly Staphylococcus, have consistently been reported to be the most common DFUs pathogens in the Western literature. ${ }^{21}$ This finding is also corroborated by our study, where $60 \%$ of the bacteria isolated are Gram-positive. In an opposite direction, recent studies performed in Mexico and Greece recorded a worrisome evolution toward a Gram-negative bacteria predominance (53\% to $57 \%$ ), even though $S$ aureus remained the most commonly isolated pathogen ( $22 \%$ to $27 \%)^{22,23}$

Similarly to the previously mentioned Portuguese studies, Gram-positive bacteria were predominant within our microbiological background, with $S$ aureus being the most 
common isolate. Nevertheless, our prevalence of Gramnegatives isolates $(40 \%)$ is superior than the one reported by Mendes et al (19\%). This disagreement can be due to the distinctive features of the study populations, since ours corresponds to moderate-to-severe DFIs that required hospitalization, whereas in the Mendes et al's study, only $34.7 \%$ of the cultures were performed on inpatients. Additionally, $40.8 \%$ of the bacteria identified in our population were collected from cultures performed on DFIs of patients previously followed in the outpatient clinic, that is, long-standing ulcers with numerous visits to health care facilities to undergo optimal dressing treatments. Moreover, the difference between the percentage of Gram-negative bacteria found in our OG $(51 \%)$ and in the EG $(32.4 \%)$ is easily understandable and explained by the previously described factors.

Considering the ISDA guidelines and the recommendation of the Portuguese Directorate-General of Health, the choice of a broad-spectrum agent must be adjusted to its effectiveness, to the specific microbiological background, and correspondent AST. A direct comparison between piperacillin/tazobactam and imipenem/cillastin was drawn by Saltoglu et al with similar clinical outcomes. ${ }^{24}$ The ISDA recommendation is to consider imipenem/cillastin when ESBL-producing pathogens are suspected, which only encompass $2.4 \%$ of our isolates. ${ }^{10}$ Overall safety and efficacy rates are similar when comparing piperacillin/tazobactam with ampicillin/sulbactam, albeit the latter has shown decreased efficacy against $P$ aeruginos $a .{ }^{25}$ The studies performed comparing piperacillin/tazobactam with ertapenem stated that both drugs have comparable clinical results in treating DFI. ${ }^{26,27}$ Nevertheless, ertapenem lacks activity against $P$ aeruginosa and Enterococcus spp. ${ }^{28-30}$ Takimoto et al published, in 2017, a review of the effectiveness of piperacillin/tazobactam in the treatment of complicated skin and soft tissues infections, which includes DFI, supporting its use in severe infection, over ertapenem or moxifloxacin, due to the emergence of resistant organisms to the late antibiotics. ${ }^{31}$ Piperacillin/tazobactam also recorded the lowest antibiotic resistance rate within the Gram-negative pathogens (including $P$ aeruginosa) in a Greek DF unit study. ${ }^{22}$

Considering the AST data and the comparison established between recommended empiric antibiotics (Tables 2 and 3), there are some highlights to address. Excluding MRSA, Gram-positive bacteria represented $45 \%$ of the pathogens recorded $(n=56)$, and almost all of them were sensitive to amoxicillin/clavulanic acid and piperacillin/ tazobactam, with the exception of one isolate of Enterococcus faecalis. MRSA accounted for $15.2 \%(\mathrm{n}=19)$ of the isolates, and its prevalence was similar between the 2 discriminated groups ( $\mathrm{EG}=15 \%, \mathrm{OG}=15.7 \%$ ), which means that this pathogen was isolated in 19 out of 87 cultures performed $(22 \%)$. This finding underlines the importance of evaluating the risk factors for community-acquired MRSA in order to add, in empiric antibiotic regimens, anti-MRSA coverture. Fermenter Gram-negative bacteria $(\mathrm{n}=30$, $24 \%$ ) recorded a prohibitive resistance rate to amoxicillin/ clavulanic acid (73\%), contrasting to ertapenem $(0 \%)$ and piperacillin/tazobactam (13\%). The prevalence of $P$ aeruginosa $(\mathrm{n}=15,12 \%)$ and $E$ faecalis $(\mathrm{n}=15,12 \%)$ explains the difference between the global coverage rate of ertapenem $(56.8 \%)$ and piperacillin/tazobactam (72.8\%). Within the scientific community, there is still debate about the pathogenic role of these 2 bacteria, with some authors claiming that it does not have a significant impact in the clinical outcome. ${ }^{32}$ On the other hand, according to Zhang et al, lower healing rates and higher amputation rates were correlated with isolated multidrug resistant $P$ aeruginosa, which enhances its pathogenic role. ${ }^{33}$ In our pool of microbiological cultures, the exclusive growth of $P$ aeruginosa (n $=7)$ or E faecalis $(\mathrm{n}=3)$ was verified in the presence of clinically evident moderate-to-severe infection. Henceforth, it is wise to establish a direct causal relationship and causative agents of the infectious process. Taking all facts into consideration, especially the low activity of ertapenem against these 2 bacteria and the emergence of carbapenemase-producing Klebsiella pneumoniae in Portugal, ${ }^{34,35}$ we are convicted that piperacillin/tazobactam should be the broad-spectrum antibiotic chosen in moderate-to-severe DFI. Ertapenem should be considered a second-line agent or an alternative to piperacillin/tazobactam if it is contraindicated or if it does not allow sufficient antibiotic coverage. ${ }^{34,35}$ The addition of linezolid or vancomycin to the antibiotic regimen for MRSA-specific coverage must be recommended for severe DFI. We encourage an exhaustive screening for MRSA-specific risk factors at baseline patient evaluation. Due to the MRSA low resistance rates to cotrimoxazol this antibiotic has a potential role as a therapeutic option in empiric coverage. However, the studies performed on this topic involved only mild/moderate DFI that did not require hospitalization. ${ }^{15,36} \mathrm{~A}$ well-designed, prospective, randomized, controlled trial should be performed in order evaluate the use of cotrimoxazol in moderate-to-severe DFI.

DF units are encouraged to routinely reassess the validity of their empirical antibiotic regimen, so our study is of extreme value for our practice and to provide insight of the Portuguese reality, as studies have been so far scarce. However, they are insufficient to build a national guideline. First, the study design did not measure other risk factors (peripheral arterial disease, glycemic control, previous antibiotic treatments, and others) that may influence the microbiological infective DF flora. Additionally, systematic differentiation between the severity grades, III and IV (PEDIS) or moderate and severe (ISDA), was not performed. Finally, it is impossible to draw general resistance rate conclusions based on a limited number of isolates, 
exemplified by the Gram-negative rods (Enterobacter spp $\mathrm{n}=5 ;$ E coli $\mathrm{n}=7$; and Klebsiella $\operatorname{spp} \mathrm{n}=4$ ). A longitudinal, well-designed prospective study with a multivariate analysis might be able to define risk factors-specific antibiotic regimens. Nevertheless, our data provide valuable information for antibiotic selection in a real-world scenario where limb or even life-threatening DF infections require immediate, but as targeted as possible, antibiotic treatment.

\section{Conclusions}

Gram-positive bacteria are still the main causative pathogens of DFI. Our study highlights piperacillin/tazobactam as the best first-line empirical antibiotic option to treat moderate-to-severe DFI in our Portuguese DF-referral inpatient center. Screening for MRSA-specific risk factors is mandatory and an anti-MRSA agent should be added early if necessary. Further longitudinal research is warranted to make grounds for a new Portuguese Guideline for proper DF empirical antibiotherapy.

\section{Authors' Note}

The authors declare that no patient data appear in this article.

\section{Acknowledgments}

The authors would like to thank Dr. Joana Cabete and Dr. Maria Helena Estrada from the Centro Hospitalar Universitário de Lisboa Central for reviewing this article.

\section{Declaration of Conflicting Interests}

The author(s) declared no potential conflicts of interest with respect to the research, authorship, and/or publication of this article.

\section{Funding}

The author(s) received no financial support for the research, authorship, and/or publication of this article.

\section{Ethical Approval}

The study protocol followed our institute's guidelines and was approved by the ethics committee on human research.

\section{References}

1. Mottola C, Mendes JJ, Cristino JM, Cavaco-Silva P, Tavares L, Oliveira M. Polymicrobial biofilms by diabetic foot clinical isolates. Folia Microbiol (Praha). 2016;61:35-43. doi:10.1007/s12223-015-0401-3

2. International Diabetes Organization. IDF Diabetes Atlas 2017. Brussels, Belgium: International Diabetes Organization; 2008.

3. Observatorio Nacional da Diabetes. Diabetes Factos E Numeros-O Ano de 2015. http://spd.pt/images/OND/ DFN2015.pdf. Accessed March 15, 2019.
4. Cabete J, Martins de Carvalho F, Moniz L, Pinto M, Neves J, Alves PC. Microbiological profile and antibiotic susceptibility patterns of organisms isolated from diabetic foot ulcers in a Portuguese Hospital [in Portuguese]. Rev Port Cir Cardiotorac Vasc. 2011;18:53-60.

5. Katz DE, Friedman ND, Ostrovski E, et al. Diabetic foot infection in hospitalized adults. J Infect Chemother. 2016;22:167173. doi:10.1016/j.jiac.2015.12.007

6. Lipsky BA. Diabetic foot infections: current treatment and delaying the "post-antibiotic era." Diabetes Metab Res Rev. 2016;32(suppl 1):246-253.

7. Mendes JJ, Marques-costa A, Vilela C, et al. Clinical and bacteriological survey of diabetic foot infections in Lisbon. Diabetes Res Clin Pract. 2011;95:153-161. doi:10.1016/j. diabres.2011.10.001

8. Olid AS, Solà I, Barajas-nava LA, et al. Systemic antibiotics for treating diabetic foot infections (review). Cochrane Database Syst Rev. 2015;(9):CD009061. doi:10.1002/14651858. CD009061.pub2

9. Lipsky BA, Berendt AR, Cornia PB, et al. 2012 Infectious Diseases Society of America clinical practice guidelines for the diagnosis and treatment of diabetic foot infections. $J \mathrm{Am}$ Podiatr Med Assoc. 2013;103:2-7.

10. Lipsky BA, Berendt AR, Cornia PB, et al; Infectious Diseases Society of America. 2012 Infectious Diseases Society of America clinical practice guideline for the diagnosis and treatment of diabetic foot infections. Clin Infect Dis. 2012;54:e132-e173. doi:10.1093/cid/cis346

11. Chuan F, Tang K, Jiang P, Zhou B, He X. Reliability and validity of the perfusion, extent, depth, infection and sensation (PEDIS) classification system and score in patients with diabetic foot ulcer. PLoS One. 2015;10:e0124739. doi:10.1371/ journal.pone.0124739

12. Mendes JJ, Neves J. Diabetic foot infections: current diagnosis and treatment. J Diabet Foot Complicat. 2012;4:26-45.

13. Feng SH, Chu YJ, Wang PH, Jun X, Min D, Li XM. Risk factors and gene type for infections of MRSA in diabetic foot patients in Tianjin, China. Int J Low Extrem Wounds. 2013;12:106-112. doi:10.1177/1534734613489991

14. Reveles KR, Duhon BM, Moore RJ, Hand EO, Howell CK. Epidemiology of methicillin-resistant Staphylococcus aureus diabetic foot infections in a large academic hospital: implications for antimicrobial stewardship. PLoS One. 2016;11:0161658. doi:10.1371/journal.pone.0161658

15. Eleftheriadou I, Tentolouris N, Argiana V, Jude E, Boulton AJ. Methicillin-resistant Staphylococcus aureus in diabetic foot infections. Drugs. 2010;70:1785-1797. doi:10.2165/11538070-000000000-00000

16. Bus SA, van Netten JJ, Lavery LA, et al; International Working Group on the Diabetic Foot. IWGDF Guidance on the prevention of foot ulcers in at-risk patients with diabetes. Diabetes Metab Res Rev. 2016;32(suppl 1):16-24. doi:10.1002/dmrr.2696

17. Uçkay I, Gariani K, Dubois-Ferrière V, Suvà D, Lipsky BA. Diabetic foot infections: recent literature and cornerstones of management. Curr Opin Infect Dis. 2016;29:145-152. doi:10.1097/QCO.0000000000000243

18. Spichler A, Hurwitz BL, Armstrong DG, Lipsky BA. Microbiology of diabetic foot infections : from Louis Pasteur 
to "crime scene investigation." $B M C$ Med. 2015;13:2. doi:10.1186/s12916-014-0232-0

19. George M. Organização de cuidados, prevenção e tratamento do Pé Diabético. Lisboa, Portugal: Direção Geral Saúde Port; 2011.

20. Barbosa AL, Carvalho R, Carvalho AC. Evaluation of the efficacy of a diabetic foot infection empirical antibiotic therapy protocol: a retrospective inpatient study. Rev Port Diabetes. 2016;11:14-17.

21. Hatipoglu M, Mutluoglu M, Turhan V, et al. Causative pathogens and antibiotic resistance in diabetic foot infections: a prospective multi-center study. J Diabetes Complications. 2016;30:910-916. doi:10.1016/j.jdiacomp.2016.02.013

22. Demetriou M, Papanas N, Panagopoulos P, Panopoulou M, Maltezos E. Antibiotic resistance in diabetic foot soft tissue infections : a series from Greece. Int J Low Extrem Wounds. 2017;16:255-259. doi:10.1177/1534734617737640

23. Sánchez-Sánchez M, Cruz-Pulido WL, Bladinieres-Cámara E, Alcalá-Durán R, Rivera-Sánchez G, Bocanegra-García V. Bacterial prevalence and antibiotic resistance in clinical isolates of diabetic foot ulcers in the Northeast of Tamaulipas, Mexico. Int J Low Extrem Wounds. 2017;16:129-134. doi:10.1177/1534734617705254

24. Saltoglu N, Dalkiran A, Tetiker T, et al. Piperacillin/tazobactam versus imipenem/cilastatin for severe diabetic foot infections: a prospective, randomized clinical trial in a university hospital. Clin Microbiol Infect. 2010;16:1252-1257. doi:10.1111/j.1469-0691.2009.03067.x

25. Harkless L, Boghossian J, Pollak R, et al. An open-label, randomized study comparing efficacy and safety of intravenous piperacillin/tazobactam and ampicillin/sulbactam for infected diabetic foot ulcers. Surg Infect (Larchmt). 2005;6:27-40.

26. Lipsky BA, Armstrong DG, Citron DM, Tice AD, Morgenstern DE, Abramson MA. Ertapenem versus piperacillin/tazobactam for diabetic foot infections (SIDESTEP): prospective, randomised, controlled, double-blinded, multicentre trial. Lancet. 2005;366:1695-1703. doi:10.1016/ S0140-6736(05)67694-5

27. Xu ZR, Ran XW, Xian Y, et al. Ertapenem versus piperacillin/tazobactam for diabetic foot infections in China: a phase 3, multicentre, randomized, double-blind, active-controlled, non-inferiority trial. J Antimicrob Chemother. 2016;71:16881696. doi:10.1093/jac/dkw004
28. Schubert S, Dalhoff A. Activity of moxifloxacin, imipenem, and ertapenem against Escherichia coli, Enterobacter cloacae, Enterococcus faecalis, and Bacteroides fragilis in monocultures and mixed cultures in an in vitro pharmacokinetic/ pharmacodynamic model simulating concentrations in the human pancreas. Antimicrob Agents Chemother. 2012;56:6434-6436. doi:10.1128/AAC.00872-12

29. Livermore DM, Carter MW, Bagel S, et al. In vitro activities of ertapenem (MK-0826) against recent clinical bacteria collected in Europe and Australia. Antimicrob Agents Chemother. 2001;45:1860-1867. doi:10.1128/AAC.45.6. 1860-1867.2001

30. Zhanel GG, Wiebe R, Dilay L, et al. Comparative review of the carbapenems. 2007;67:1027-1052.

31. Takimoto K, Wang Q, Suzuki D, Katayama M, Hayashi Y. Clinical efficacy of piperacillin/tazobactam in the treatment of complicated skin and soft tissue infections. Expert Opin Pharmacother. 2017;18:1027-1034. doi:10.1080/14656566.2 017.1341491

32. Roberts AD, Simon GL. Diabetic foot infections: the role of microbiology and antibiotic treatment. Semin Vasc Surg. 2012;25:75-81. doi:10.1053/j.semvascsurg.2012.04.010

33. Zhang J, Chu Y, Wang P, et al. Clinical outcomes of multidrug resistant Pseudomonas aeruginosa infection and the relationship with type iii secretion system in patients with diabetic foot. Int J Low Extrem Wounds. 2014;13:205-210. doi:10.1177/1534734614545878

34. European Centre for Disease Prevention and Control Summary of the latest data on antibiotic consumption in EU: 2017. https://ecdc.europa.eu/en/publications-data/summary-latest-data-antibiotic-consumption-eu-2017. Published November 15, 2017. Accessed March 15, 2019.

35. European Centre for Disease Prevention and Control. Summary of the latest data on antibiotic resistance in the European Union. https://ecdc.europa.eu/en/publications-data/ summary-latest-data-antibiotic-resistance-european-union. Published November 15, 2017. Accessed March 15, 2019.

36. Stein GE, Throckmorton JK, Scharmen AE, et al. Tissue penetration and antimicrobial activity of standard- and highdose trimethoprim/sulfamethoxazole and linezolid in patients with diabetic foot infection. $J$ Antimicrob Chemother. 2013;68:2852-2858. doi:10.1093/jac/dkt267 